\title{
Evaluation of Responses in MTL Model Excited from Multiple Stochastic Sources
}

\author{
Lubomír Brančík, and Edita Kolářová
}

\begin{abstract}
The paper presents a technique for simulation of stochastic responses and their statistical characteristics in multiconductor transmission lines (MTL). The method follows a theory of stochastic differential equations (SDE) and relevant numerical techniques for their solution. The MTL's model, formed via generalized $\Pi$ sections in cascade, is designed to cover various situations at stochastic and/or deterministic excitations. In this way both the noisy pulses driving MTL's terminal ports and effects of possible unwanted disturbances along the MTL's wires are allowed to be simulated. First the state-variable method is applied to derive a deterministic description, then voltage stochastic variations are incorporated to define the vector linear SDE. To obtain the characteristics of stochastic responses, firstly the set of trajectories is statistically processed, while a weak stochastic backward Euler scheme, consistent with the Itô stochastic calculus, is applied. Finally, a method of direct calculation of variances, based on the solution of relevant Lyapunov-like differential equations, is used with advantage. All the simulations were performed in the Matlab language environment.
\end{abstract}

Keywords-Multiconductor transmission line, state variable, stochastic differential equation, stochastic source.

\section{INTRODUCTION}

The techniques based on stochastic differential equations (SDE) find their place in many fields of engineering where stochastic influences are relevant to be considered [1]-[3]. In the electrical engineering SDE approach has been used in many various fields, see at least [4]-[6]. An interesting application is to simulate stochastic responses in systems with distributed parameters like e.g. transmission lines (TL), often used in high-speed electronic systems [7]-[9]. Both internal structural noises and possible external disturbances can be covered. In former papers the SDE approach was applied for TL systems in which the external drivings were only possible from TLs' terminating ports [10]-[12]. In [13] a possibility to consider multiple external random sources is proposed, however, only for the single TLs. In this paper an extension of this approach is done towards MTL's model as shown in Fig. 1, where the excitations are allowed in any node. Such a model can be used to simulate effects of unwanted random disturbances along the MTL wires, for

Manuscript received November 22, 2015, revised January 23, 2016. This work was supported by Czech Science Foundation under grant 15-18288S "Research of Signal Integrity at High-Speed Interconnects". For research, infrastructure of the SIX Center was used.

L. Brančík is with the Department of Radio Electronics, Brno University of Technology, Technicka 12, 61600 Brno, Czech Republic (phone: +420 541146563; fax:+420 541146596; e-mail: brancik@feec.vutbr.cz).

E. Kolářová is with the Department of Mathematics, Brno University of Technology, Technicka 8, 61600 Brno, Czech Republic (e-mail: kolara@feec.vutbr.cz)

doi: $10.11601 /$ ijates.v5i1.145 example [14]. To formulate a basic SDE system description a state-variable method is applied on the MTL's generalized RLCG-based model, and generalized Thévenin equivalents are utilized to join the sources to respective nodes [15]. In Fig. 1, non-deterministic voltage vectors are marked by asterisks, but various combinations are allowed. A task is to find the statistical characteristics of the stochastic responses: the expectation and the standard deviation. Hence a weak stochastic backward Euler scheme is used for the numerical solution of the SDEs, which utilizes two-point distributed random variables as initial stochastic processes [2], [3]. In addition to [14], direct calculation of variances of stochastic responses is performed, utilizing Lyapunov-like differential and algebraic matrix equations [16], [17]. All methods were programmed in the Matlab language environment.

\section{Deterministic Model Formulation}

We will consider MTL, nonuniform in general, defined by its length $l$ and per-unit-length (p.u.l.) matrices $\mathbf{L}_{0}(x), \mathbf{C}_{0}(x)$, $\mathbf{R}_{0}(x)$ and $\mathbf{G}_{0}(x)$ of the order $n \times n$, with $n$ as a number of active conductors. In the MTL $m$-sectional lumpedparameter model in Fig. 1, the voltage vectors $\mathbf{v}_{k}$, $k=1, \ldots, m+1$, and the current vectors $\mathbf{i}_{k}, k=1, \ldots, m$, are the $n \times 1$ vectors of the state variables in view. The model lumped parameters are set by matrices $\mathbf{L}_{k}=\mathbf{L}_{0}\left(x_{k}+\Delta x / 2\right) \Delta x$, $\mathbf{C}_{k}=\mathbf{C}_{0}\left(x_{k}\right) \Delta x, \mathbf{R}_{k}=\mathbf{R}_{0}\left(x_{k}+\Delta x / 2\right) \Delta x$ and $\mathbf{G}_{k}=\mathbf{G}_{0}\left(x_{k}\right) \Delta x$, with $\Delta x=l / m$ [10]. The border shunt elements, $\mathbf{C}_{1}, \mathbf{G}_{1}$, and $\mathbf{C}_{m+1}$, $\mathbf{G}_{m+1}$, are taken half-size to ensure the model to be a cascade connection of generalized $\Pi$ networks of the same type. The current vectors of external sources are determined by $\mathbf{i}_{\mathrm{i} k}=\mathbf{G}_{\mathrm{i} k}\left(\mathbf{v}_{\mathrm{i} k}-\mathbf{v}_{k}\right)$, where the internal Thévenin matrices are supposed as regular, i.e. $\mathbf{G}_{\mathrm{i} k}=\mathbf{R}_{\mathrm{i} k}^{-1}$ exists.

A state-variable description of the MTL model in Fig. 1, first with all the parameters deterministic (excitation voltage vectors $\mathbf{v}_{\mathrm{i} k}{ }^{*}$ replaced by $\mathbf{v}_{\mathrm{i} k}$ ) can be written as

$$
\mathbf{M} \frac{d \mathbf{x}(t)}{d t}=-(\mathbf{H}+\mathbf{P}) \mathbf{x}(t)+\mathbf{P u}(t)
$$

with terms as follows. The vector of state variables

$$
\mathbf{x}(t)=\left[\begin{array}{l}
\mathbf{v}_{\mathrm{C}}(t) \\
\mathbf{i}_{\mathrm{L}}(t)
\end{array}\right]
$$

contains $n(2 m+1)$ elements grouped to $n \times 1$ column vectors, namely $\mathbf{v}_{\mathrm{C}}(t)$ holds $m+1$ vectors of state voltages and $\mathbf{i}_{\mathrm{L}}(t)$ holds $m$ vectors of state currents. The matrix

$$
\mathbf{M}=\operatorname{diag}(\mathbf{C}, \mathbf{L})
$$

holds the block diagonal matrices $\mathbf{C}=\operatorname{diag}\left(\mathbf{C}_{1}, \mathbf{C}_{2}, \ldots, \mathbf{C}_{m+1}\right)$, $\mathbf{L}=\operatorname{diag}\left(\mathbf{L}_{1}, \mathbf{L}_{2}, \ldots, \mathbf{L}_{m}\right)$, and zero matrices $\mathbf{0}$. The matrix 


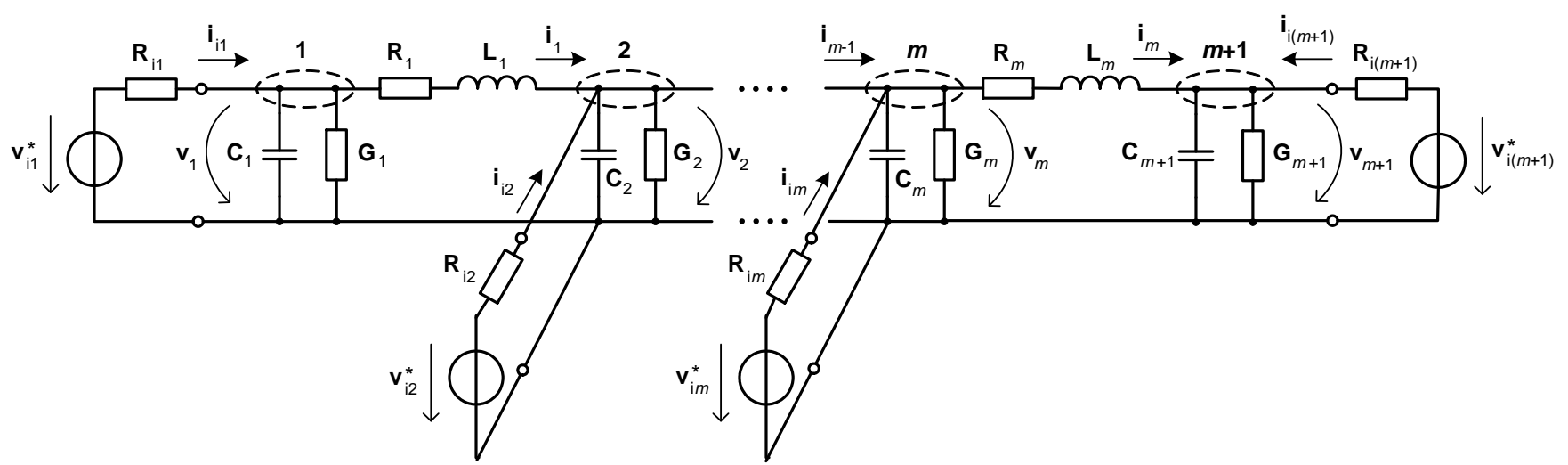

Fig. 1. Schematic representation of MTL model excited from stochastic sources.

$$
\mathbf{H}=\left[\begin{array}{cc}
\mathbf{G} & \mathbf{E} \\
-\mathbf{E}^{\mathrm{T}} & \mathbf{R}
\end{array}\right]
$$

is similarly created by the matrices $\mathbf{G}=\operatorname{diag}\left(\mathbf{G}_{1}, \mathbf{G}_{2}, \ldots, \mathbf{G}_{m+1}\right)$, $\mathbf{R}=\operatorname{diag}\left(\mathbf{R}_{1}, \mathbf{R}_{2}, \ldots, \mathbf{R}_{m}\right)$, and a block matrix $\mathbf{E}$ containing the identity matrices $\pm \mathbf{I}$ and zero matrices $\mathbf{0}$, see [10] for details. The matrix

$$
\mathbf{P}=\left[\begin{array}{ll}
\mathbf{Y}_{\mathrm{I}} & \mathbf{0} \\
\mathbf{0} & \mathbf{0}
\end{array}\right]
$$

depends on external circuits and contains the block diagonal $\operatorname{submatrix} \mathbf{Y}_{\mathrm{I}}=\operatorname{diag}\left(\mathbf{G}_{\mathrm{i} 1}, \mathbf{G}_{\mathrm{i} 2}, \ldots, \mathbf{G}_{\mathrm{i}(m+1)}\right)$. The drive vector

$$
\mathbf{u}(t)=\left[\begin{array}{c}
\mathbf{v}_{\mathrm{I}}(t) \\
\mathbf{0}
\end{array}\right]
$$

contains the subvector $\mathbf{v}_{\mathrm{I}}(t)=\left[\mathbf{v}_{\mathrm{i} 1}{ }^{\mathrm{T}}(t), \mathbf{v}_{\mathrm{i} 2}{ }^{\mathrm{T}}(t), \ldots, \mathbf{v}_{\mathrm{i}(m+1)}{ }^{\mathrm{T}}(t)\right]^{\mathrm{T}}$. If we introduce denotations

$$
\mathbf{A}=-\mathbf{M}^{-1}(\mathbf{H}+\mathbf{P}), \quad \mathbf{B}=\mathbf{M}^{-1} \mathbf{P}
$$

then (1) can be written

$$
\frac{d \mathbf{x}(t)}{d t}=\mathbf{A} \mathbf{x}(t)+\mathbf{B u}(t)
$$

with a formal solution

$$
\mathbf{x}(t)=e^{\mathbf{A} t} \mathbf{x}(0)+\int_{0}^{t} e^{\mathbf{A} \cdot(t-\tau)} \mathbf{B u}(\tau) d \tau
$$

if $t=0$ is chosen as a beginning of the time interval of interest.

\section{MODEL WITH STOCHASTIC EXCITATIONS}

In this section the Itô stochastic calculus [1] is applied to get responses to the input sources influenced by stochastic processes. Instead of $\mathbf{v}_{\mathrm{i} k}(t)$, used in the deterministic solution above, stochastic versions of the voltage vectors are used as

$$
\mathbf{v}_{\mathrm{i} k}^{*}(t)=\mathbf{v}_{\mathrm{i} k}(t)+\boldsymbol{\alpha}_{k} \xi_{k}(t)
$$

$k=1,2, \ldots, m+1$, where $\xi_{k}(t)=\left[\xi_{k 1}(t), \xi_{k 2}(t), \ldots, \xi_{k n}(t)\right]^{\mathrm{T}}$ are noise vectors whose elements $\xi_{k l}(t)$ represent white noise processes (related to the $l$-th wire of the MTL, and the $k$-th section of its model), and $\boldsymbol{\alpha}_{k}=\operatorname{diag}\left(\alpha_{k 1}, \alpha_{k 2}, \ldots, \alpha_{k n}\right)$ are constant matrices whose elements $\alpha_{k l}$ express intensities of respective noises. Then, taking into account (6) and (10), (8) can be rewritten as

$$
\frac{d \mathbf{x}(t)}{d t}=\mathbf{A x}(t)+\mathbf{B}(\mathbf{u}(t)+\delta \mathbf{u}(t))
$$

where

$$
\delta \mathbf{u}(t)=\left[\begin{array}{c}
\delta \mathbf{v}_{\mathrm{I}}(t) \\
\mathbf{0}
\end{array}\right]=\left[\begin{array}{c}
\boldsymbol{\alpha} \xi(t) \\
\mathbf{0}
\end{array}\right]
$$

with a noise intensity matrix $\boldsymbol{\alpha}=\operatorname{diag}\left(\boldsymbol{\alpha}_{1}, \boldsymbol{\alpha}_{2}, \ldots, \boldsymbol{\alpha}_{m+1}\right)$, and a stochastic processes vector $\xi(t)=\left[\xi_{1}{ }^{\mathrm{T}}(t), \xi_{2}{ }^{\mathrm{T}}(t), \ldots, \xi_{m+1}{ }^{\mathrm{T}}(t)\right]^{\mathrm{T}}$.

A method of converting the differential equation (11) on its stochastic version lies in multiplying it by $d t$, and replacing all the products $\xi_{k l}(t) d t$ by $d W_{k l}(t)$, with $W_{k l}(t)$ designating Wiener processes [1]. By this the vector linear SDE with additive noises is formulated

$$
d \mathbf{X}(t)=(\mathbf{A X}(t)+\mathbf{B u}(t)) d t+d \mathbf{b}_{\mathrm{W}}(t)
$$

where $d \mathbf{b}_{\mathrm{W}}(t)$ arises from (11) and (12), namely

$$
\begin{gathered}
d \mathbf{b}_{\mathrm{W}}(t)=\mathbf{B}\left[\begin{array}{c}
\boldsymbol{\alpha} d \mathbf{W}(t) \\
\mathbf{0}
\end{array}\right]=\mathbf{B} \sum_{k=1}^{m+1} \sum_{l=1}^{n}\left[\begin{array}{c}
\boldsymbol{\alpha}^{(p)} \\
\mathbf{0}
\end{array}\right] d W_{k l}(t)= \\
=\sum_{k=1}^{m+1} \sum_{l=1}^{n} \mathbf{b}^{(p)} d W_{k l}(t)
\end{gathered}
$$

with $\boldsymbol{\alpha}^{(p)}$ denoting the $p$-th column of the noise intensity matrix $\alpha$, while $p=n(k-1)+l$, and $d \mathbf{W}(t)$ containing differentials of Wiener processes.

It is common to mark stochastic solutions by capital letters to distinguish them from deterministic ones, like $\mathbf{X}(t)$ vs. $\mathbf{x}(t)$ in (13) and (8), respectively. Considering a multidimensional Itô formula and marking in (14), the SDE (13) has a solution

$$
\begin{aligned}
\mathbf{X}(t) & =e^{\mathbf{A} t} \mathbf{X}(0)+\int_{0}^{t} e^{\mathbf{A}(t-\tau)} \mathbf{B u}(\tau) d \tau+ \\
& +\sum_{k=1}^{m+1} \sum_{l=1}^{n} \int_{0}^{t} e^{\mathbf{A}(t-\tau)} \mathbf{b}^{(p)} d W_{k l}(\tau)
\end{aligned}
$$

with $\mathbf{b}^{(p)}$ defined in (14), while $p=n(k-1)+l$. The right terms in (15) are called Itô integrals. The solution $\mathbf{X}(t)$ is a random process and for its expectation we have for $t>0$

$$
E[\mathbf{X}(t)]=e^{\mathbf{A} t} E[\mathbf{X}(0)]+\int_{0}^{t} e^{\mathbf{A}(t-\tau)} \mathbf{B u}(\tau) d \tau
$$

as expectations of the Itô integrals are zero. It is evident that for a constant initial value $\mathbf{X}(0)$ the expectation of a stochastic solution agrees with the deterministic solution (9). 


\section{ApPlication OF Numerical Methods}

\section{A. Stochastic Backward Differentiation Formulae}

When considering a numerical technique from the theory of ordinary differential equations (ODE) some care is needed as according to the definition of the Itô integral a stochastic integral must always be evaluated at lower endpoint of the discretization interval. To solve SDEs we can utilize the stochastic backward Euler scheme [2], consistent with the Itô stochastic calculus, and sufficiently stable for higherorder circuits represented by the MTL's model.

Let us consider the solution $\mathbf{X}(t)$ on the interval $t_{0} \leq t \leq T$, with respect to an equidistant discretization $t_{r}=t_{0}+r h$, where $h=\left(T-t_{0}\right) / N=t_{r+1}-t_{r}, r=0, \ldots, N-1$, and with the discretization of the Wiener processes $\Delta W_{k l}^{(r)}=W_{k l}\left(t_{r+1}\right)-W_{k l}\left(t_{r}\right)=\int_{t_{r}}^{t_{r+1}} d W_{k l}(\tau)$. To use a stochastic numerical scheme, random increments of the Wiener processes should be generated as the independent Gaussian random variables, while $E\left[\Delta \mathrm{W}_{k l}^{(r)}\right]=0$ and $E\left[\left(\Delta \mathrm{W}_{k l}^{(r)}\right)^{2}\right]=h$. Subsequently, the stochastic backward Euler scheme for solving (13) can be written as

$$
\begin{gathered}
\mathbf{X}^{(r+1)}=(\mathbf{I}-\mathbf{A} h)^{-\mathbf{1}}\left(\mathbf{X}^{(r)}+\mathbf{B} \mathbf{u}^{(r+1)} h+\Delta \mathbf{b}_{\mathrm{W}}^{(r)}\right)= \\
=(\mathbf{I}-\mathbf{A} h)^{-\mathbf{1}}\left(\mathbf{X}^{(r)}+\mathbf{B} \mathbf{u}^{(r+1)} h+\sum_{k=1}^{m+1} \sum_{l=1}^{n} \mathbf{b}^{(p)} \Delta W_{k l}^{(r)}\right)
\end{gathered}
$$

where $p=n(k-1)+l$. The solution (17) is one with a strong convergence, and it is useful when the individual stochastic trajectories are important. Our problem does not require good pathwise approximations but only the approximations of the expectations $\mu_{k l}=E\left[X_{k l}\right]$ and the standard deviations $\sigma_{k l}=\sqrt{ } E\left[\left(X_{k l}-\mu_{k l}\right)^{2}\right]$ of the SDE solutions. Therefore, we can apply a weak version of (17), where the increments of the Wiener processes $\Delta W_{k l}^{(r)}$ are replaced by simplier, two-point distributed random variables $\Delta \widehat{W}_{k l}^{(r)}$, while the probability $P\left(\Delta \widehat{W}_{k l}^{(r)}= \pm \sqrt{ } h\right)=1 / 2$. The numerical efficiency of the weak scheme is generally better then of the strong one [2], [3].

\section{B. Estimates of Statistical Characteristics}

For the vector linear SDE with additive noises, like (13), a resultant stochastic process $\mathbf{X}(t)$ has the multivariate normal distribution [2], [3]. We are not going to study any correlations, so we can use the usual procedure to determine confidence intervals for individual items $X_{k l}(t)$, based on the Student's $t$-distribution [2], [18]. Taking into account a sole item of interest, and marking $X_{k l}^{(r)}=X_{k l}\left(t_{r}\right)$, as in (17), the sample mean and sample standard deviation are respectively determined via all $J$ realizations by the formulae [19]

$$
\begin{gathered}
\bar{X}_{k l}^{(r)}=\frac{1}{J} \sum_{j=1}^{J} X_{k l, j}^{(r)} \\
s_{k l}^{(r)}=\sqrt{\frac{1}{J-1} \sum_{j=1}^{J}\left(X_{k l, j}^{(r)}-\bar{X}_{k l}^{(r)}\right)^{2}}
\end{gathered}
$$

for $r=0, \ldots, N-1$ to cover whole time interval. Then, based on a Student's $t$-distribution with $J-1$ degrees of freedom, a two-sided $100(1-\alpha) \%$ confidence interval (related to individual trajectories) is determined as

$$
\left(\bar{X}_{k l}^{(r)}-t_{1-\alpha / 2, J-1} s_{k l}^{(r)}, \bar{X}_{k l}^{(r)}+t_{1-\alpha / 2, J-1} s_{k l}^{(r)}\right)
$$

In case we need confidence interval for sample mean it can be got from (20) by replacing $s_{k l}^{(r)}$ with $s_{k l}{ }^{(r)} / \sqrt{ } J$.

\section{Expectation and Variance Direct Computations}

As was mentioned above, when considering in (16) constant initial conditions $\mathbf{X}(0)$, the expectation $\boldsymbol{\mu}(t)=E[\mathbf{X}(t)]$ of the stochastic responses $\mathbf{X}(t)$ follows respective deterministic solution (9), namely

$$
\frac{d \boldsymbol{\mu}(t)}{d t}=\mathbf{A} \boldsymbol{\mu}(t)+\mathbf{B u}(t)
$$

To enable forming confidence intervals the variances of stochastic processes are needed. As is known, the variances $D_{k}(t)=\sigma_{k}^{2}(t)=\operatorname{var}\left(X_{k}(t)\right), k=1, \ldots, n(2 m+1)$, with $\sigma_{k}(t)$ as the standard deviation, are diagonal elements of the covariance matrix $\boldsymbol{\Sigma}(t)=\operatorname{cov}(\mathbf{X}(t))$ of respective random vector $\mathbf{X}(t)=\left[X_{1}(t), \ldots, X_{n(2 m+1)}(t)\right]^{\mathrm{T}}$. It is valid [19]

$$
\begin{aligned}
\boldsymbol{\Sigma}(t) & =E\left[(\mathbf{X}(t)-\boldsymbol{\mu}(t))(\mathbf{X}(t)-\boldsymbol{\mu}(t))^{\mathrm{T}}\right]= \\
& =E\left[\mathbf{X}(t) \mathbf{X}(t)^{\mathrm{T}}\right]-\boldsymbol{\mu}(t) \boldsymbol{\mu}(t)^{\mathrm{T}}
\end{aligned}
$$

If we now designate $\mathbf{Q}(t)=E\left[\mathbf{X}(t) \mathbf{X}(t)^{\mathrm{T}}\right]$, then taking into account (13) and (14), and following the theory in [3], $\mathbf{Q}(t)$ satisfies the Lyapunov-like matrix ODE

$$
\begin{gathered}
\frac{d \mathbf{Q}(t)}{d t}=\mathbf{A} \mathbf{Q}(t)+\mathbf{Q}(t) \mathbf{A}^{\mathrm{T}}+\mathbf{B} \mathbf{u}(t) \boldsymbol{\mu}(t)^{\mathrm{T}}+ \\
+\boldsymbol{\mu}(t)(\mathbf{B u}(t))^{\mathrm{T}}+\sum_{k=1}^{m+1} \sum_{l=1}^{n} \mathbf{b}^{(p)} \mathbf{b}^{(p) \mathrm{T}}
\end{gathered}
$$

with $p=n(k-1)+l$, and the expectation $\boldsymbol{\mu}(t)$ in (21). To solve (21), a backward Euler scheme is used, with a time step $h$, as

$$
\boldsymbol{\mu}^{(r+1)}=(\mathbf{I}-\mathbf{A} h)^{-1} \cdot\left(\boldsymbol{\mu}^{(r)}+\mathbf{B} \mathbf{u}^{(r+1)} h\right)
$$

To solve (23), the same numerical scheme leads to the Lyapunov-like matrix equation [20]

$$
\begin{gathered}
(\mathbf{I}-\mathbf{A} h) \mathbf{Q}^{(r+1)}-\mathbf{Q}^{(r+1)} \mathbf{A}^{\mathrm{T}} h=\mathbf{Q}^{(r)}+ \\
+\left(\mathbf{B} \mathbf{u}^{(r+1)} \boldsymbol{\mu}^{(r+1) \mathrm{T}}+\boldsymbol{\mu}^{(r+1)}\left(\mathbf{B} \mathbf{u}^{(r+1)}\right)^{\mathrm{T}}+\sum_{k=1}^{m+1} \sum_{l=1}^{n} \mathbf{b}^{(p)} \mathbf{b}^{(p) \mathrm{T}}\right) h
\end{gathered}
$$

where $\boldsymbol{\mu}^{(r+1)}$ is given by (24), with the same step $h$, and being resolved for $\mathbf{Q}^{(r+1)}$, e.g. by using an alternating-directionimplicit (ADI) iteration [17]. Then, designating $\mathbf{V}=\mathbf{I}-\mathbf{A} h$, $\mathbf{W}=-\mathrm{A}^{\mathrm{T}} h$, and $\mathbf{F}^{(r+1)}$ as the right side of (25), we solve in each time step, $r=0,1, \ldots$, a matrix equation

$$
\mathbf{V} \mathbf{Q}^{(r+1)}+\mathbf{Q}^{(r+1)} \mathbf{W}=\mathbf{F}^{(r+1)}
$$

by ADI iterations

$$
\begin{aligned}
& \left(\mathbf{V}+p_{j} \mathbf{I}\right) \mathbf{Q}_{j-1 / 2}^{(r+1)}=\mathbf{F}^{(r+1)}-\mathbf{Q}_{j-1}^{(r+1)}\left(\mathbf{W}-p_{j} \mathbf{I}\right) \\
& \mathbf{Q}_{j}^{(r+1)}\left(\mathbf{W}+q_{j} \mathbf{I}\right)=\mathbf{F}^{(r+1)}-\left(\mathbf{V}-q_{j} \mathbf{I}\right) \mathbf{Q}_{j-1 / 2}^{(r+1)}
\end{aligned}
$$

$J=1,2, \ldots$, and $\mathbf{Q}_{0}{ }^{(r+1)}=\mathbf{Q}^{(r)}$. The coefficients $p_{j}$ and $q_{j}$ can be used to improve the speed of convergence, in following examples, they are set to $p_{j}=q_{j}=1$. 
Due to a normality of the resultant stochastic processes, the $100 \cdot(1-\alpha) \%$ confidence interval is given by

$$
\left(\mu_{k l}^{(r)}-u_{1-\alpha / 2} \sigma_{k l}^{(r)}, \mu_{k l}^{(r)}+u_{1-\alpha / 2} \sigma_{k l}^{(r)}\right)
$$

with $u_{1-\alpha / 2}$ as a quantile of a standard normal distribution.

\section{Simulation Examples in Matlab}

\section{A. Introductory Example - Single $T L$}

To better explain crossing to multiconductor transmission line (MTL) models, an introductory single TL, i.e. $n=1$, will first be considered. In this case, all variables are scalars instead of column vectors and square matrices. Besides, a possibility to utilize two-point random variables as initial stochastic process to estimate confidence interval will be verified, as well as computation of variances via Lyapunovlike ODEs and corresponding numerical technique.

Thus, let us consider TL of the length $l=0.2 \mathrm{~m}$, with perunit-length parameters $L_{0}=494.6 \mathrm{nH} / \mathrm{m}, C_{0}=62.8 \mathrm{pF} / \mathrm{m}$, $R_{0}=0.1 \Omega / \mathrm{m}$ and $G_{0}=0.1 \mathrm{~S} / \mathrm{m}$ [21], whose model is composed of $m=128$ parts. First, all voltages are set zero, i.e. $v_{\mathrm{i} k}^{*}(t)=0, k=2, \ldots, m+1$, except $v^{*}{ }_{\mathrm{i} 1}(t)=v_{\mathrm{S}}(t)+\alpha_{1} \xi_{1}(t)$, with $\alpha_{1}=10^{-6}$, which excites a left port, while $v_{\mathrm{S}}(t)$ is a $1 \mathrm{~V}$ trapezoidal pulse with $0.5 \mathrm{~ns}$ rise/fall times and $3 \mathrm{~ns}$ width. A source resistance $R_{\mathrm{i} 1}=R_{\mathrm{S}}=5 \Omega$, and a load resistance $R_{\mathrm{i} 129}=\mathrm{R}_{\mathrm{L}}=10 \mathrm{k} \Omega$. As no excitation sources are now connected along the TL model, remaining resistances $R_{\mathrm{i} k} \rightarrow \infty, k=2, \ldots, m$. Results of these simulations are in Figs. 2 and 3 for both terminal ports.
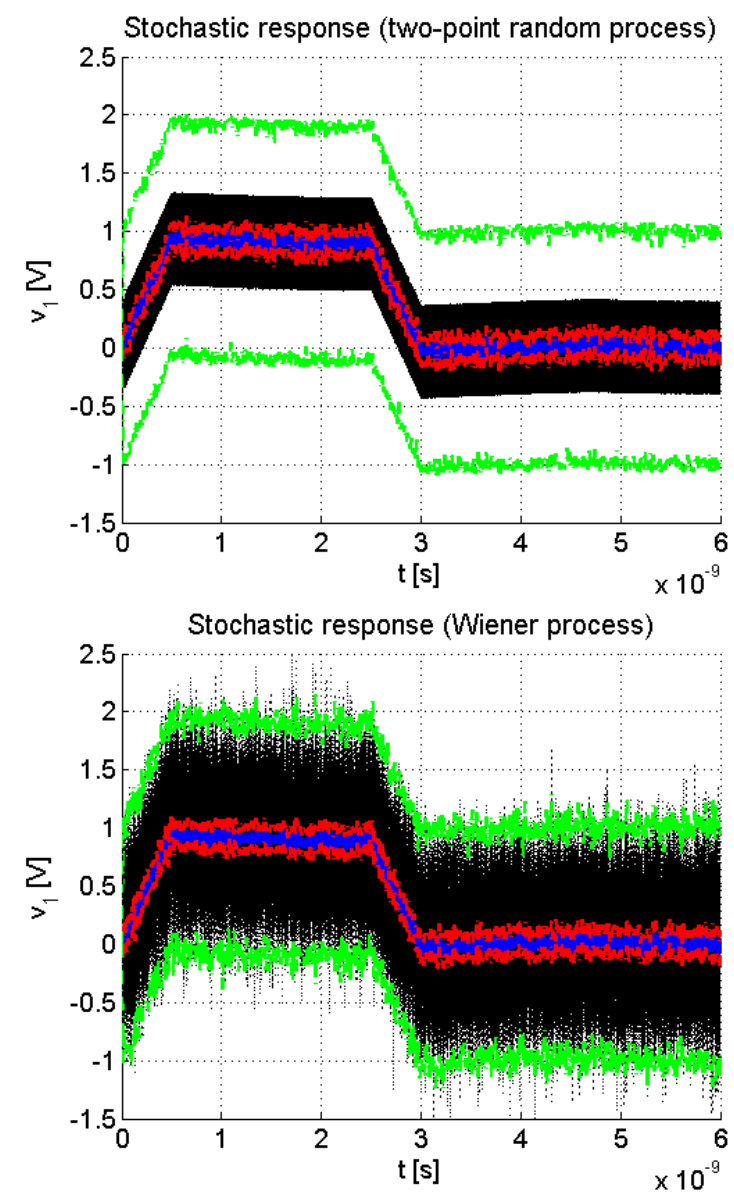

Fig. 2. Comparison of different initial stochastic processes (input port).
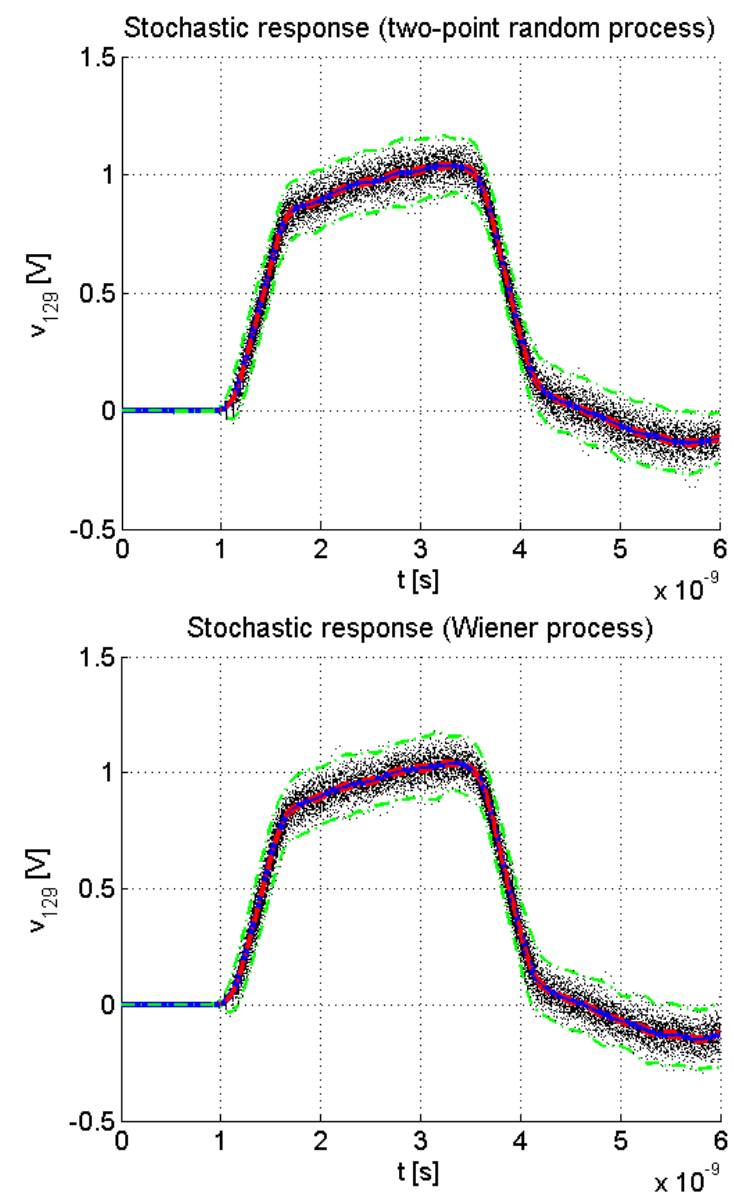

Fig. 3. Comparison of different initial stochastic processes (output port).
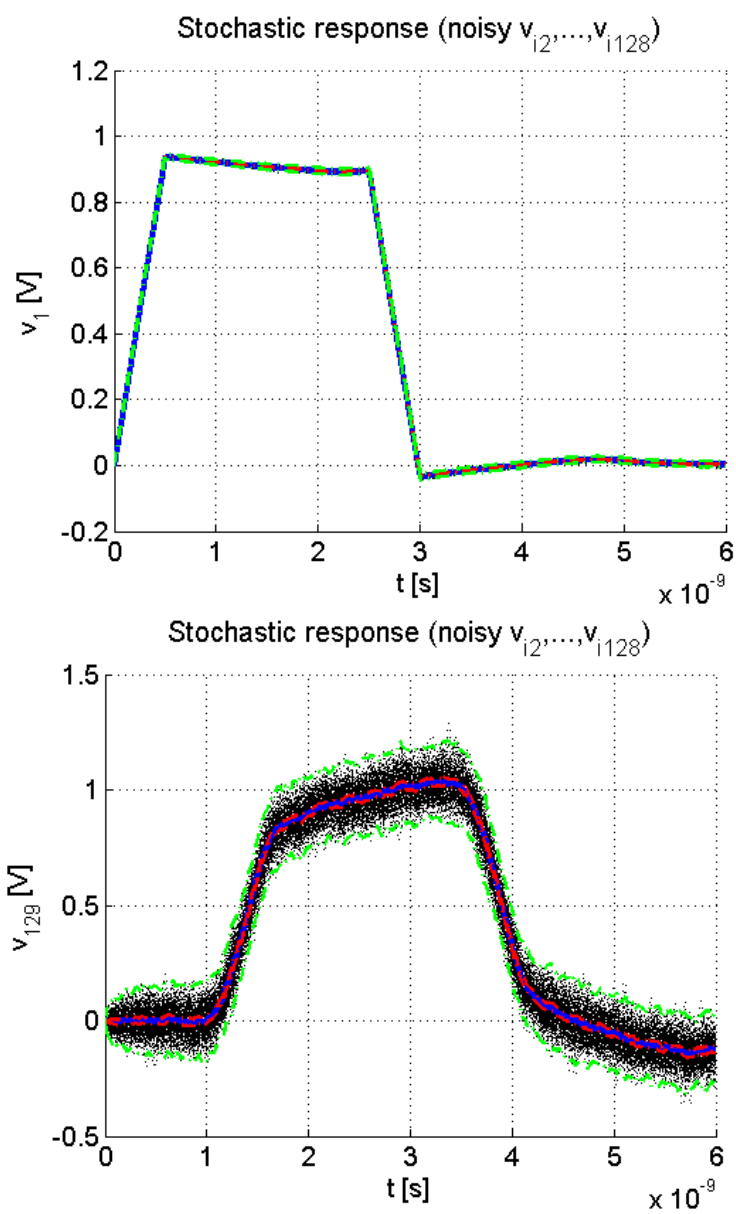

Fig. 4. Responses influenced by random disturbances along the TL. 
At the model's left port in Fig. 2, differences at individual stochastic trajectories (black) are strong due to nearness of the excitation source. However, it is also evident, that confidence intervals are very similar (here $99 \%$, computed from 100 samples, red for sample means, green for individual samples). At the right port in Fig. 3, there is no notable difference in a character of stochastic trajectories. In light of estimate of confidence interval both the processes are well exchangeable, $\mathrm{cp}$. theory in [2].

The other example in Fig. 4 considers an input voltage $v^{*}{ }_{\mathrm{i} 1}(t)=v_{\mathrm{S}}(t)$ as deterministic, with the same resistances at the left and right ports, however, the remaining sources are stochastic, namely $v^{*}{ }_{\mathrm{i} k}(t)=\alpha_{k} \xi_{k}(t)$, with $\alpha_{k}=10^{-3}$, and the resistances $R_{\mathrm{i} k}=1 \mathrm{M} \Omega, k=2, \ldots, m$. As excitation voltages are exclusively stochastic, i.e. without deterministic parts, random disturbances along the TL can e.g. be covered in this way.

\section{B. Simulation of Simple MTL}

As an example of the simulation of stochastic responses on the MTL, the uniform $(2+1)$-conductor TL terminated with resistive elements is considered in Fig. 5 [14].

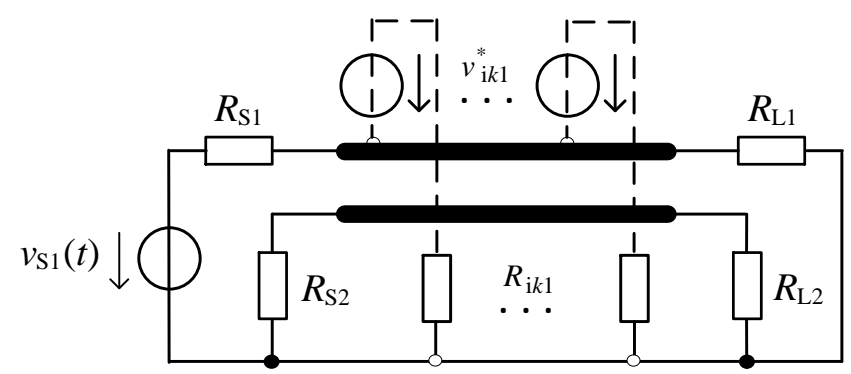

Fig. 5. MTL system with stochastic excitations $v^{*}{ }_{i k 1}$.

The MTL has the length $l=0.4 \mathrm{~m}$, and is characterized by p.u.1. parameters [21]

$$
\begin{aligned}
\mathbf{R}_{0} & =\left[\begin{array}{cc}
0.1 & 0.02 \\
0.02 & 0.1
\end{array}\right] \Omega / \mathrm{m}, \mathbf{L}_{0}=\left[\begin{array}{cc}
494.6 & 63.3 \\
63.3 & 494.6
\end{array}\right] \mathrm{nH} / \mathrm{m} \\
\mathbf{G}_{0} & =\left[\begin{array}{cc}
0.1 & -0.01 \\
-0.01 & 0.1
\end{array}\right] \mathrm{S} / \mathrm{m}, \mathbf{C}_{0}=\left[\begin{array}{cc}
62.8 & -4.9 \\
-4.9 & 62.8
\end{array}\right] \mathrm{pF} / \mathrm{m}
\end{aligned}
$$

The MTL's model is composed of $m=50$ sections, and due to the uniformity, its parameters follow (30) multiplied by $l / m$. The parameters of Thévenin models at terminal ports, $\mathbf{R}_{\mathrm{S}}=\operatorname{diag}\left(R_{\mathrm{S} 1}, R_{\mathrm{S} 2}\right), \quad \mathbf{R}_{\mathrm{L}}=\operatorname{diag}\left(R_{\mathrm{L} 1}, R_{\mathrm{L} 2}\right), \quad \mathbf{v}_{\mathrm{S}}(t)=\left[v_{\mathrm{S} 1}(t), 0\right]^{\mathrm{T}}$, and $\mathbf{v}_{\mathrm{L}}(t)=[0,0]^{\mathrm{T}}$, correspond to those of the MTL model in Fig. 1, i.e. $\mathbf{R}_{\mathrm{i} 1}, \mathbf{R}_{\mathrm{i} 51}, \mathbf{v}_{\mathrm{i} 1}(t)$, and $\mathbf{v}_{\mathrm{i} 51}(t)$, respectively, while $R_{\mathrm{S} 1}=R_{\mathrm{S} 2}=5 \Omega, \quad R_{\mathrm{L} 1}=R_{\mathrm{L} 2}=10 \mathrm{k} \Omega, \quad$ and the excitation voltage $v_{\mathrm{S} 1}(t)=\sin ^{2}\left(\pi t / 2 \cdot 10^{-9}\right)$ if $0 \leq t \leq 2 \cdot 10^{-9} \mathrm{~s}$, and $v_{\mathrm{S} 1}(t)=0$ otherwise. The MTL's first wire is excited from stochastic sources, as is schematically sketched by dash lines in Fig. 5. The voltage vectors $\mathbf{v}_{\mathrm{i} k}^{*}(t)=\left[v_{\mathrm{i} k 1}^{*}(t), 0\right]^{\mathrm{T}}$, with $v_{\mathrm{i} k 1}^{*}(t)=\alpha_{k 1} \xi_{k 1}(t)$, and noise intensities $\alpha_{k 1}=10^{-3}$, with zero deterministic components, and conductance matrices $\mathbf{G}_{\mathrm{i} k}=\mathbf{R}_{\mathrm{i} k}{ }^{-1}=\operatorname{diag}\left(R_{\mathrm{i} k 1}{ }^{-1}, 0\right), \quad R_{\mathrm{i} k 1}=1 \mathrm{M} \Omega, \quad k=2, \ldots, m . \quad$ In short, the MTL is driven from a deterministic source on its left port, into the first wire, while stochastic disturbances act along the MTL's first wire.

The statistical characteristics of stochastic responses for the MTL's terminating ports are shown in Fig. 6, computed from sets of $J=100$ samples via (18)-(20): the sample means by solid blue lines, the $99 \%$ confidence intervals by dashed red lines (for the means) and dashed green lines (for the samples). For practical usage, just estimates of moments of stochastic processes are most often needed.
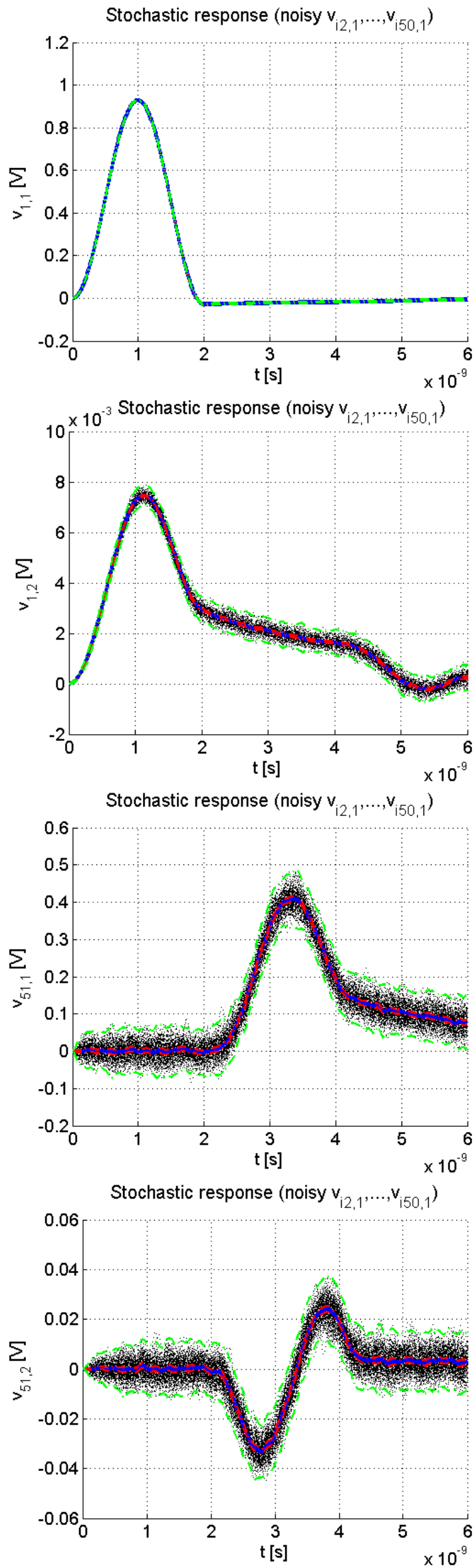

Fig. 6. Statistical characteristics of MTL's stochastic responses. 
In Fig. 7, there are the results corresponding to Fig. 6, however, the confidence interval is calculated directly based on the Lyapunov-like matrix equation (25).
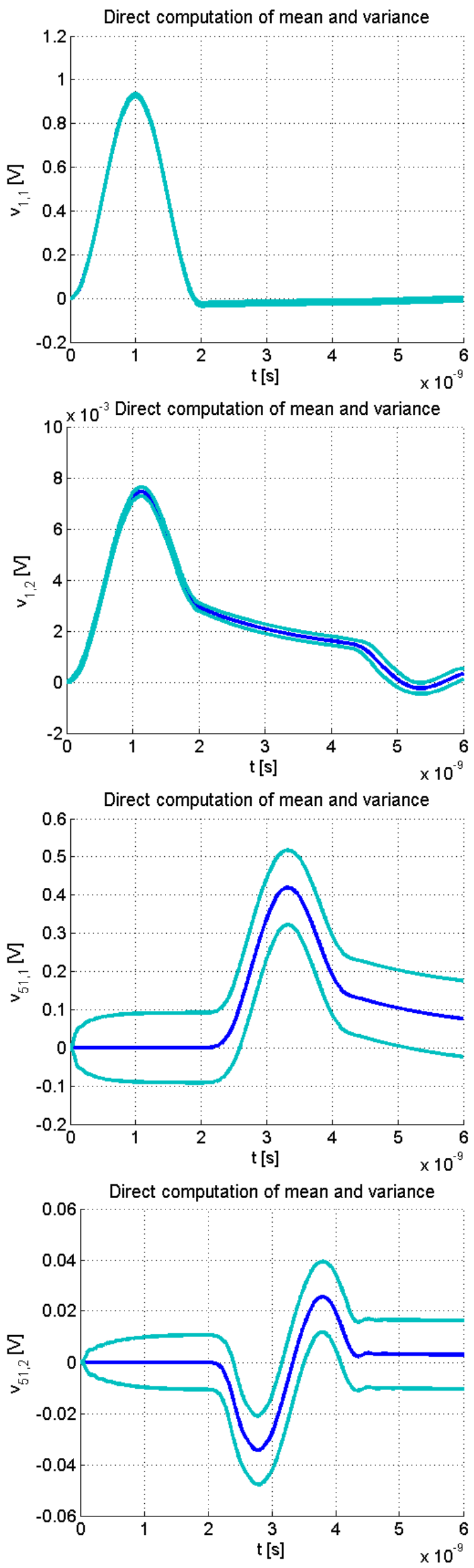

Fig. 7. Direct calculation of variances for MTL's stochastic responses.
As can be seen from Fig. 7 the confidence intervals well correspond to those in Fig. 6, but there was no need to repeatedly solve SDEs themselves. This approach is well applicable just for additive noises considered here because the normal distribution of stochastic responses is kept, and consequently, the inverse normal distribution function can be directly applied to get respective confidence intervals.

\section{CONCLUSION}

The paper dealt with a method for simulation of statistical characteristics of stochastic responses at MTLs' model with excitations from multiple stochastic and/or deterministic sources. Beyond the previously published works, where only MTL's terminal ports were allowed for the excitations, the method extends it to arbitrary nodes along the MTL model, that can advantageously be used when unwanted disturbances along the wires arise. Besides two versions of initial stochastic processes were applied and the results compared - the Wiener process, needed if individual stochastic trajectories are studied, and simplified two-point random variable process, sufficient when only the moments of stochastic processes are of our interest. It was verified on the single transmission line simulation that the confidence intervals calculated via both the methods agree very well. Finally a method utilizing direct computation of variances of stochastic processes, based on the solution of a covariance matrix, was verified and compared with statistical methods. For this purpose the Lyapunov-like differential equation has been developed in relation to the MTL's model, and proper numerical technique based on an alternating-directionimplicit iteration method verified. It should be noticed that just this method seems to be most appropriate for practical purposes, to evaluate dispersions of random processes with a defined confidence level. All the computations were performed in the Matlab language, utilizing a sparse matrix notation with advantages for saving RAM and CPU time.

Methods based on the SDE theory can be interesting and efficient alternatives to other probabilistic techniques used in field of electronic circuits simulations, like e.g. widespread Monte Carlo [22], which is highly time-consuming method. In systems with distributed parameters, frequently in interconnects at high-speed digital/analog circuits, a polynomial-chaos expansion method is also often used to evaluate manufacturing random changes [23], [24], or various methods following sensitivities computations can be considered [25], [26]. It should be noticed that the SDEs approach is applicable for the analysis of further parameters, related to a physical structure of interconnects, and leading to SDEs with a multiplicative noise [10], [27]. Similarly, for more complex hybrid systems, stochastic differential algebraic equations (SDAE) can be utilized [6], [28].

\section{REFERENCES}

[1] B. Øksendal, Stochastic Differential Equations, An Introduction with Applications. New York: Springer-Verlag, 2000.

[2] P. Kloeden, E. Platen, and H. Schurz, Numerical Solution of SDE Through Computer Experiments. New York: Springer-Verlag, 1997.

[3] L. Arnold, Stochastic Differential Equations: Theory and Applications. Malabar: Krieger Publishing, 1992.

[4] D. Ham, A. Hajimiri, "Complete noise analysis for CMOS switching mixers via stochastic differential equations," in Proc. IEEE Custom Integrated Circuits Conference, Orlando, 2000, pp. 439-442. 
[5] A. Zjajo, Q. Tang, M. Berkelaar, J. P. de Gyvez, A. Di Bucchianico, N. van der Meijs, "Stochastic analysis of deep-submicrometer CMOS process for reliable circuits designs," IEEE Transactions on Circuits and Systems I: Regular Papers, vol. 58, no. 1, 2011, pp. 164-175.

[6] K. Wang, M. L. Crow, "Numerical simulation of stochastic differential algebraic equations for power system transient stability with random loads," in Proc. 2011 IEEE Power and Energy Society General Meeting, San Diego, 2011, pp. 1-8.

[7] M. P. LI, Jitter, Noise, and Signal Integrity at High-Speed. Upper Saddle River: Prentice Hall, 2007.

[8] C. K. Cheng, J. Lillis, S. Lin, N. Chang, Interconnect Analysis and synthesis. New York: John Wiley \& Sons, 2000.

[9] C. R. Paul, Analysis of Multiconductor Transmission Lines. New York: John Wiley \& Sons, 2008.

[10] L. Brančík, E. Kolářová, "Stochastic differential equations approach in the analysis of MTLs with randomly varied parameters," in Proc. 19th IEEE International Conference on Electronics, Circuits, and Systems ICECS'2012, Sevilla, Spain, 2012, pp. 725-728.

[11] L. Brančík, E. Kolářova, "Simulation of higher-order electrical circuits with stochastic parameters via SDEs," Advances in Electrical and Computer Engineering, vol. 13, no. 1, 2013, pp. 17-22.

[12] L. Brančík, E. Kolářová, "Simulation of stochastic responses at multiconductor transmission lines with fluctuating parameters," in Proc. 23rd International Conference Radioelektronika'2013, Pardubice, Czech Republic, 2013, pp. 61-64.

[13] L. Brančík, E. Kolářová, "Time-domain simulation of transmission line models with multiple stochastic excitations," in Proc. 24th International Conference Radioelektronika'2014, Bratislava, Slovakia, 2014, pp. 1-4.

[14] L. Brančík, E. Kolářová, "Multiconductor transmission line models excited from multiple stochastic sources," in 37th International Conference on Telecommunications and Signal Processing TSP'14," Berlin, Germany, 2014, pp. 282-286.

[15] L. Brančík, "Time and Laplace-domain methods for MTL transient and sensitivity analysis," COMPEL: The International Journal for Computation and Mathematics in Electrical and Electronic Engineering, vol. 30, no. 4, 2011, pp. 1205-1223.

[16] G. H. Golub, C. F. Van Loan, Matrix computations. Baltimore: Johns Hopkins Univ. Press, 1996.

[17] E. Wachspress, Iterative solution of the Lyapunov matrix equation. Appl. Math. Lett., vol. 1, 1998, pp. 87-90.
[18] E. Kolářova,"Statistical estimates of stochastic solutions of RL electrical circuits," in Proc. the IEEE International Conference on Industrial Technology ICIT'06, Mumbai, India, 2006, pp. 2546-2550.

[19] M. G. Bulmer, Principles of Statistics. Dover Publications, Revised edition, 1979.

[20] L. Brančík, E. Kolářova, "Confidence intervals at multiconductor transmission lines with stochastic excitations," in 22nd European Conference on Circuit Theory and Design ECCTD'2015, Trondheim, Norway, 2015, p. 1-4.

[21] S. Lum, M. S. Nakhla, and Q. J. Zhang, "Sensitivity analysis of lossy coupled transmission lines," IEEE Transactions on Microwave Theory and Techniques, vol. 39, no. 12, 1991, pp. 2089-2099.

[22] D. P. Kroese, T. Taimre, and Z. I. Botev, Handbook of Monte Carlo methods. New York: John Wiley \& Sons, 2011.

[23] D. Spina, F. Ferranti, T. Dhaene, et al. "Variability analysis of multiport systems via polynomial-chaos expansion," IEEE Transactions on Microwave Theory and Techniques, vol. 60, no. 8, 2012, pp. 2329-2338.

[24] D. Vande Ginste, D. De Zutter, D. Deschrijver, T. Dhaene, P Manfredi, and F. Canavero, "Stochastic modeling-based variability analysis of on-chip interconnects," IEEE Transactions on Components, Packaging and Manufacturing Technology, vol. 2, no. 7, 2012, pp. 1182-1192.

[25] A. Wardzinska, W. Bandurski W., "Step response sensitivity to RLC parameters of VLSI interconnect," in Proc. International Conference on Signals and Electronic Systems ICSES'10, Gliwice, Poland, 2010, pp. 297-300.

[26] H. Qinshu, L. Xinen, and X. Shifu, "A fast approximate method for parametric probabilistic sensitivity estimation," in Proc. 3rd International Conference on Advanced Computer Theory and Engineering ICACTE'10, Chengdu, China, 2010, pp. V3-349-V3-351.

[27] L. Brančík, E. Kolářová, "Variance assesment at transmission lines with randomly varying parameters via SDE theory," in Proc. 16th International Conference on Computer as a Tool EUROCON'2015, Salamanca, Spain, 2015, pp. 287-291.

[28] L. Brancik, "Simulation of hybrid MTL systems with random parameters based on stochastic DAEs," in Proc. 21st European Conference on Circuit Theory and Design ECCTD'2013, Dresden, Germany, 2013, pp. 1-4. 